
\title{
R Reserarch Suare \\ IgG seroprevalence against SARS-CoV-2 in a cohort of 449 non-hospitalized, high-risk exposure individuals.
}

Patricia Torres Martínez

Empireo

Paula Diaque García

Empireo

María Rubio Salas

Empireo

Raquel Rodríguez Sánchez

Empireo

Felipe García

Empireo https://orcid.org/0000-0002-4099-0120

Miguel Ángel Llamas

Empireo

Paula Saz-Leal

Inmunotek

CArlos del Fresno ( $\sim$ carlosdelfresno@empireo.es )

Empireo

Article

Keywords: COVID-19, SARS-CoV-2, seroprevalence, high-risk exposure

Posted Date: August 18th, 2020

DOI: https://doi.org/10.21203/rs.3.rs-53747/v1

License: (c) (1) This work is licensed under a Creative Commons Attribution 4.0 International License.

Read Full License 


\section{Abstract}

COVID-19 pandemic caused by SARS-CoV-2 led the Spanish government to impose a national lockdown in an attempt to control the spread of the infection. Mobility restrictions but also the requirement of a medical prescription to gain access to serological testing for COVID-19 were included among the measures. Under this scenario, between April 15th to June 15th, 2020, we performed a seroprevalence observational study including 449 individuals that fulfill prescription requirements: manifesting COVID-19 compatible symptoms, being in contact with a COVID-19 confirmed case or belonging to essential occupations including healthcare workers, firefighters or public safety personnel such as police. Importantly, none of the participants was hospitalized. Altogether, we studied this specific, non-commonly addressed cohort for SARS-CoV-2 seroprevalence, uncovering intrinsic features of great demographic interest. The overall rate of IgG seropositivity was $33.69 \%$ (95\% Cl: $29.27-38.21)$. This seroprevalence was comparable between different occupations performed by the participants. However, contacts with confirmed cases associated positively with IgG+ results, with stronger correlation if being a household member. The number of symptoms also correlated positively with IgG+ prevalence. Ageusia/anosmia, pneumonia and cutaneous manifestations were the top-three symptoms that most strongly associated with IgG+ seroprevalence. However, while pneumonia and cutaneous manifestations were barely present in our cohort, fever, ageusia/anosmia and asthenia were the most frequently symptoms described within IgG+ subjects. Therefore, our data illustrate how specific cohorts display heterogeneous characteristics that should be taken into account when identifying population seroprevalence against SARS-CoV-2 and key defining symptoms for COVID-19.

\section{Introduction}

The humanity is facing one of the most severe challenges in the last times due to the SARS-CoV-2 coronavirus infection. This infection was early identified in January 2020 as the etiologic agent for a pneumonia outbreak detected in Wuhan City, China, in December 2019. The resulting Coronavirus Disease-19 (COVID-19) has quickly spread all around the world, being declared as global pandemic by the World Health Organization (WHO) in March $2020^{1}$, only three months after the first cases were detected.

Since its first appearance, the scientific world has struggled to collect data from affected patients in an attempt to understand the pathophysiology of this infection. Of note, most of the infected individuals are asymptomatic ${ }^{2}$ and indeed, this is a critical feature to explain the rapid dissemination of SARS-CoV- $2^{3}$. In symptomatic patients, clinical manifestations of COVID-19 range from mild to moderate upper respiratory tract illness, leading to either recovery or severe pneumonia and eventual death ${ }^{4,5}$. Fever, dry cough and dyspnea are among the most common symptoms ${ }^{6}$.

Of note, most of these studies have been performed analyzing hospitalized patients, usually ranging between mild to severe diagnosis 7,8 . However, as stated before, most of the infected population does not show symptoms or at least, not enough symptoms to seek for clinical attention. This particular 
characteristics of COVID-19 have made difficult to accurately ascertain the actual rate of SARS- CoV-2 infection and create a portfolio of common clinical features.

National governments are trying to screen their populations in an unbiased manner trying to identify and isolate infected people and to determine the actual rate of infection ${ }^{9}$. This information could be really informative as in an scenario of massive spreading of the virus, herd immunity could be achieved, that could help to control the community infection ${ }^{10}$. In this sense, the Spanish Ministry of Health has performed a massive seroprevalence study called ENE-COVID, analyzing the presence of anti-SARS-CoV-2 specific IgG nationwide, including more than 60,000 people chosen randomly ${ }^{11}$. Seroprevalence for the entire country was determined in the range of $3.7 \%$ to $6.2 \%$, depending on the technique used in the test, either immunochromatographic or chemiluminescent immunoassay ${ }^{11}$. Interestingly, the distribution of the seroprevalence was not homogenous along the country, with some provinces showing increased seropositivity for anti-SARS-CoV-2 IgG. As a such, Madrid province reported a seroprevalence between $11.3 \%$ and $11.5 \%{ }^{11}$.

Seroprevalence studies have been also performed in the context of health care workers (HCWs), as they are the front-line defense against the infection. These studies show that the seroprevalence is slightly higher in this high-risk exposure personnel, with data ranging from $9.3 \%^{12}$ to $10.3 \%^{13}$ in two studies performed in Catalonia (Spain), where the regional seroprevalence according to the national study range between $6.8 \%$ and $7.0 \%{ }^{11}$. Therefore, the study of particular populations provides unique information about the impact of SARS- CoV-2 infection under specific demographic features, revealing clues to better understand the pathophysiology of COVID-19.

In here we provide data about anti SARS-CoV-2 IgG seroprevalence in a large cohort of 449 subjects from Madrid province, which either reported COVID-19 compatible symptoms or high-risk exposure to the infection, but importantly, that did not required hospital assistance despite showing symptoms. We believe that the analysis of this population and its intrinsic features harbors an invaluable piece of information about the seroprevalence of a high-risk exposure population to the SARS-CoV-2 infection in a non-hospital-related environment. Of note, the cohort also included essential occupations such as healthcare professionals, allowing a comparison analysis in this key subpopulation group.

\section{Results}

\section{Baseline characteristics}

Starting from 729 eligible individuals, a total of 449 were included in the study after excluding subjects who declined to participate and unable to contact. Among them, $55.68 \%$ were male and $44.32 \%$ female (Table 1). The mean age of participants was 45.74 years (95\% Cl: $44.38-47.07)$. Sixteen percent declared themselves as smokers and $22.94 \%$ reported having comorbidities (Table 1 ). Regarding work occupation, $9.58 \%, 4.01 \%$ and $6.46 \%$ were healthcare workers (HCWs), firefighters or worked for preserving public safety, respectively, all of them considered as essential occupations during Spanish 
lockdown. The rest of the individuals did not belong to these specific professions. As said before, due to mobility restrictions imposed during the lockdown, all the subjects resided in Madrid area, $33.18 \%$ of them in Madrid city.

Forty-six participants (10.24\%) were previously tested by PCR, with $28.26 \%$ positive results among them. Sixty-two percent of the participants reported contact with a COVID-19 confirmed case, being either a household member $(48.39 \% ; 30.07 \%$ of the total) or a non-cohabitating person $(51.61 \% ; 32.07 \%$ of the total). Eventually, $69.49 \%$ of the included individuals reported COVID-19 compatible symptoms, which is also an inclusion criterion for the study (Table 1). The average time after the end of those symptoms to perform the test was 42.56 days ( $95 \% \mathrm{Cl}$ : $39.90-45.23$ ).

Importantly, despite these data related to the manifestation of symptoms, none of the admitted participants was hospitalized, indicating a specific feature of the studied population.

\section{Seroprevalence of IgG antibodies against SARS-CoV-2}

One hundred and fifty-one participants were seropositive for IgG against SARS-CoV-2, corresponding to a seroprevalence of $33.69 \%$ (95\% Cl: 29.27 - 38.21). Sex, age, presence of comorbidities and location of residence did not show any statistically significant association with seroprevalence (Table 2). However, the odds of being seropositive were lower in smoker participants (OR: $0.49,95 \% \mathrm{Cl} 0.27-0.89, \mathrm{p}=0.021$ ) (Table 2). Notably, regarding occupation, no significant differences in seroprevalence were found between any of the specific occupations or overall essential professions and the other participants. Interestingly, close to a ten percent reduction in frequencies of positive IgG were observed in $\mathrm{HCWs}(25.58 \%, 95 \% \mathrm{Cl}$ : 13.52 - 41.17, OR:0.63, ns) and public

safety personnel $(24.14 \%, 95 \% \mathrm{Cl}: 10.30-43.54, \mathrm{OR}: 0.58, \mathrm{~ns})$ when compared to other occupations (Table 2).

A significant association was expectedly found between self-reported positive PCR and IgG seropositivity (92.31\%, 95\% Cl: 63.97 - 99.81, OR: 29.98, p<0.001) (Table 3). Seroprevalence was significantly higher in those individuals who reported any contact with a confirmed COVID-19 case compared to those without contact (OR: $1.93,95 \% \mathrm{Cl}: 1.26-2.941, \mathrm{p}=0.003)$. Among those having a positive contact, IgG seroprevalence was greater if this was a household member $(48.15 \%, 95 \% \mathrm{Cl}$ : $39.47-56.41, \mathrm{OR}$ : $2.83)$ compared to a non-cohabitating contact $(29.86 \%, 95 \%$ Cl: $22.53-38.04$, OR: 1.30$)(p<0.001$, Table

The odds of being seropositive were higher in participants who reported having suffered any COVID-19compatible symptom in the previous months $41.99 \%, 95 \% \mathrm{Cl}$ : $36.45-47.68$, OR: 4.29, p<0.001). The individual symptoms more strongly associated with IgG seropositivity were (in order): ageusia/anosmia 77.36\%, 95\% Cl: 68.21 - 84.92, OR:13.57), pneumonia (75.00\%, 95\% Cl: 68.21- 84.92, OR:6.35) and cutaneous manifestations $(63.33 \%, 95 \% \mathrm{Cl}: 43.86-80.07$, OR: 3.76$)$, all of them with a $p<0.001$ in the univariable analysis (Table 3 , Figure 1a). The presence of symptoms statistically correlated with a higher 
IgG seroprevalence (OR: 4.29, $\mathrm{p}<0.001)$ even if ageusia/anosmia was excluded out of the analysis (OR: $1.83, p=0.040)$.

Increased number of symptoms was significantly associated with superior rates of seroprevalence (Table $3 ; p<0.001)$. In fact, individuals reporting 7 to 9 or even more than 10 compatible symptoms displayed around 2 -fold higher seroprevalence rates than those ranging from 1 to 6 (Table 3, Figure 1b). On the other hand, the analysis of $\lg G$ seroprevalence within age ranges revealed that only the subgroup below 20 years showed a considerable rate of asymptomatic IgG positive cases (Supp. Figure 1).

In an attempt to further explore differential features of the cohort included in this work, the frequency of the different symptoms was analyzed among IgG positive individuals (Figure 2). Fever, ageusia/anosmia and asthenia were the most frequent symptoms in descending order whereas pneumonia and cutaneous manifestations were barely represented (Figure 2). Altogether, while these data sharply contrast with the overall associations observed between symptoms and IgG seropositivity (Figure 1a), they reveal the differential features of the studied cohort and the relevance of describing specifically subpopulation groups to identify defining symptoms for COVID-19.

\section{Discussion}

Between March $15^{\text {th }}$ and June $21^{\text {st }}, 2020$, the Spanish government imposed a national lockdown in an attempt to control the extent of the COVID-19 due to SARS-CoV-2 coronavirus outbreak within the national territory ${ }^{14}$. Mobility restriction was among the exceptional measures taken, limited to the inhabiting city first and surrounding province in a second step. Only those people considered as essential personnel could move to attend their working places such as firefighters, civil servants preserving public safety and of course, healthcare workers (HCWs). Those people showing mild to moderate symptoms were asked to self-isolate at home, keeping clinical facilities for severe cases. The same preventive measures were asked for those asymptomatic population known to have been in contact with people showing COVID-19 compatible symptoms. Keeping in mind this situation, a nationwide, population-based seroepidemiological study performed between April $27^{\text {th }}$ and May $11^{\text {st }} 2020$, have shown that the seroprevalence against the SARS-CoV-2 in Spain was around five percent ${ }^{11}$. Interestingly, this seroprevalence was not uniform along the country, with areas displaying higher IgG positive rates such as Madrid province, with up to $11.5 \%$ of seroprevalence ${ }^{11}$.

Under this scenario, we performed the current study, analyzing the IgG seroprevalence against SARS-CoV2 in individuals that suffered COVID-19 compatible symptoms at least two weeks before performing the test, that knew to have been in contact with a COVID-19 diagnosed person or that belonged to an essential collective that kept working during the lockdown. Following this criteria, in a large cohort of 449 individuals, we report here an IgG seroprevalence of $33.69 \%$, much higher than the global Spanish rate 
and even than the prevalence shown in the Madrid province, where our study has been performed considering mobility restrictions.

The high IgG+ rate observed in our cohort is surely conditioned by the inclusion criteria. At the same time, this is the strength of the data provided in here. Our results illustrate how the seroprevalence rises in a population presenting symptoms and at high-risk exposure, with no further bias such as occupation. To the best of our knowledge, no studies gather such a population without discriminating by other factors. Therefore, the features included in our cohort are not dissected in population-based wide studies such as those performed in Spain ${ }^{11}$ or Switzerland ${ }^{16}$, nor in published studies addressing the seroprevalence against SARS-CoV-2 specifically in HCWs ${ }^{12,13,17-20}$.

In this sense, our data confers to HCWs an IgG seroprevalence of $25.58 \%$ (95\% IC: $13.52-41.17)$. Other studies focused in these professionals reveals rates ranging from $2 \%{ }^{18}, 7.5 \%{ }^{17,20}, 10 \%{ }^{11-13}$ to nearly $20 \%{ }^{19,21}$. Our results are in the upper range and backed by recent data from a large cohort of HCWs in a secondary teaching hospital in Madrid province ${ }^{22}$. These results are consistent with a close to two-fold higher risk for health-care workers indicated in the Spanish national seroprevalence study, as the reference population for our cohort would be the IgG+ prevalence in Madrid province, being $11.5 \%{ }^{11}$. Similarly, in two independent seroprevalence studies performed in Barcelona province, also a nearly twofold higher risk was described for HCWs, ranging between $9.3 \%{ }^{12}$ and $10.3 \%{ }^{13}$ compared with $7 \%$ in the general province population ${ }^{11}$. Also in Germany, a population-based seroprevalence study denoted a $0.94 \%$ of IgG seroprevalence in the North Rhine-Westphalia region ${ }^{23}$, while a $1.6 \%$ was reported among healthcare workers of the University Hospital Essen located in the same region ${ }^{18}$.

Interestingly, no significant differences in IgG seroprevalence were found between HCWs in our cohort and other high-risk exposure professions including firefighters or public safety personnel such as police. These data suggest that despite much attention is being payed to people working in front-line clinical assistance, other public employees are similarly high-risk exposure and they should share personal protection and training with $\mathrm{HCWs}$ to protect themselves from the coronavirus infection. Indeed, all these key professionals show the same IgG seroprevalence than people that manifested COVID-19 compatible symptoms or that established a contact with a confirmed case, as these are the other two inclusion criteria for our cohort.

A relevant factor to explain the high IgG seroprevalence against SARS- CoV-2 in the studied cohort could be the controlled period of time spent between the end of the symptoms and the test. One of the abovementioned studies was performed among HCWs exposed to COVID-19 patients; these HCWs were under quarantine for 14 days, time when their seroprevalence was analyzed ${ }^{21}$. Interestingly, the seroprevalence was in the highest range among the studies performed in HCWs $(17.14 \%)^{21}$. It could be suggested that this high rate was due to the exposition to confirmed cases, but leaving time enough to develop a robust humoral IgG response ${ }^{24}$. In fact, plasma from COVID-19 convalescent patients to be used as potential treatment against the infection, show significantly higher anti-SARS-CoV-2 titers after 
more than 42 days from symptom onset to plasma donation ${ }^{25}$. For those symptomatic subjects of our cohort, at least 14 days after the end of symptoms were required before performing the test with an average time gap of more than 40 days. Therefore, timing from the suspected contagious contact with the virus should be also considered and detailed, in an attempt to achieve a better interpretation of the seroprevalence data from diverse cohorts.

An important value of our data is to provide information about anti-SARS- CoV-2 IgG seroprevalence in a cohort where most of the subjects manifested COVID-19 compatible symptoms, but none of them required hospitalization. Early observations in hospitalized patients reported IgG+ rates as high as $80 \%$ 26,27 to even $100 \%$ in longitudinal studies ${ }^{28}$. These data seeded the hope of reaching a protective herd immunity ${ }^{29}$. However, wide nationwide studies are reporting low seroprevalence rates $11,16,30$, which are promoting pessimistic perspectives between experts regarding the achievement of such herd immunity ${ }^{31}$. Noteworthy, the seroprevalence observed in our cohort indicates that the predominance of IgGs against SARS-CoV-2 may be quite heterogeneous between populations, and heterogeneity affects massively herd immunity, modulating the percentage of infected people required to reach it ${ }^{10}$. Therefore, seroprevalence studies in controlled settings as the one performed here could be really helpful to understand population heterogeneity related to herd immunity.

Despite the test we used for this study detected both IgM and IgG against SARS-CoV-2, we decided to perform the seroprevalence study based exclusively on IgG. The main reason was the inclusion criteria of having suffered COVID-19 compatible symptoms at least, 14 days before testing, to fulfill the quarantine period established by the sanitary authorities. In line with this rationale, only $2 \% 28$ of the individuals included in our cohort was tested IgM+. Indeed, IgG detection is the base for most if not all populationbased seroprevalence studies ${ }^{11,16,30}$. However, it does not preclude the usefulness of IgM determination for early infections together with PCR as gold standard, showing a great concordance with IgG in the long term ${ }^{27}$. In this sense, and in agreement with other works ${ }^{11}$, we observed a great correlation between IgG+ seroprevalence in those individuals that self-reported a previous positive test by PCR. However, this correlation is not $100 \%$ and in fact, this result is a constant in essentially of the analyzed studies 28,32 . False positive or negative results could explain to some extent this supposed discrepancy. However, the generation of T-cell based cellular immunity could also be an underlying mechanism ${ }^{33}$.

It has been described the generation of immunogenic $\mathrm{CD} 4^{+}$and $\mathrm{CD} 8^{+}$responses against SARS-CoV-2 antigens that correlate with the presence of specific antibodies against the virus ${ }^{34,35}$. However, up to $35 \%$ of unexposed donors (without detectable anti-SARS-CoV-2 specific antibodies) showed SARS- CoV-2reactive $C D 4^{+} T$ cells, while $83 \%$ of antibody-bearing patients displayed comparable cellular responses ${ }^{36}$. Interestingly, the SARS-CoV-2-responsive $\mathrm{CD} 4^{+} \mathrm{T}$ cells from unexposed donors also responded to similar epitopes present in endemic coronaviruses $229 \mathrm{E}$ and $\mathrm{OC} 43^{36}$. Therefore, cross-reactive $\mathrm{T}$ cells might be the base for this cellular activation in the absence of humoral responses, although the dynamics of this cross-reactivity is not understood yet. As greater T cell expansion is observed in mild versus severe 
COVID-19 ${ }^{37}$, it is tempting to speculate that T cell immune responses are predominant in mild SARS-CoV2 infections.

In this sense, cellular immunity mediated by $T$ lymphocytes could also explain the presence of uninfected household members. Despite that IgG seroprevalence rises statistically among those subjects in contact with a confirmed case of SARS-CoV-2 infection ${ }^{11}$, nearly half of individuals of our cohort was seronegative for the coronavirus despite sharing home with a COVID-19 positive person. Interestingly, only mild symptoms were reported in a cohort of non-hospitalized household contacts with SARS-CoV-2 infection ${ }^{38}$, further supporting the notion that cellular immunity triggered by mild infections could underlie the lack of seroconversion. Large studies comparing mild versus severe COVID-19 cases, analyzing both cellular and humoral responses are required to fulfill this gap in our knowledge.

An intriguing data arisen from our cohort is that smokers show a reduced IgG seroprevalence against SARS-CoV-2. There could be many plausible explanations for this effect that nowadays are just speculations. It includes a direct toxic effect of smoking against the virus, impact on the hACE2 expression as the viral entry gate or a pre-inflammatory status of the pulmonary tract that helps to reduce the initial virus burden or even reduction in the production of antibodies. In any case, a large observational study enrolling nearly 150,000 participants supports that the SARS-CoV-2 infection is reduced by half among current smokers ${ }^{39}$.

As one of the criteria to be included in our study is the presence of compatible symptoms, it allows to study the relationship between their development and the seroprevalence in the studied population. Notably, there is a nice positive correlation between the number of presented symptoms and the antiSARS-CoV-2 IgG seroprevalence. This would suggest that the strength of the physical manifestations due to COVID-19 reinforces the development of antibodies against the virus ${ }^{40}$, but also that many isolated symptoms are not specific enough for the development of COVID-19, but this lack of specificity is attenuated by the combination of non-specific manifestations.

In any case, it is interesting to note that the correlation of some symptoms with the anti-SARS-CoV-2 IgG seroprevalence is much stronger than others. This supports the notion that COVID-19 is manifested with some sort of symptomatic specificity, that might help to identify and isolate infected individuals in an potential scenario of shortage of diagnostic tests. In this line, the development of ageusia/anosmia, pneumonia and cutaneous manifestations highly correlate with IgG seroprevalence, ranging between almost $80 \%$ and more than $60 \%$ when any of these symptoms appears. This data indicate that these three clinical indicators could be considered as clear determinants for the diagnosis of COVID-19 41,42.

In sharp contrast, when considering the symptoms developed among IgG+ participants in the studied cohort, it is notorious that pneumonia, cutaneous manifestations and even dyspnea are among the less frequent presented symptoms. This fact signifies that depending on the intrinsic features of the participants, some symptoms are more or less indicative of COVID-19. The studied cohort in particular, gather a population of high-risk exposure and symptomatic individuals that did not required 
hospitalization and therefore, it was unlikely to have a clinical diagnosis of pneumonia, dyspnea or even cutaneous manifestations that require skilled medical personnel to detect them. Consequently, it could be proposed the presence of ageusia/anosmia plus fever as the most accurate indicative symptoms for COVID-19 in the general population without the need of medical assistance ${ }^{43}$. The appearance of both signs could motivate a self-isolation and to contact health authorities.

In summary, the data presented in this work illustrate the IgG seroprevalence against SARS-CoV-2 in a symptomatic and high-risk exposure large population. This seroprevalence was nearly two-fold higher than what has been described in a population-based, nationwide study for the same geographic area. This relative high seroprevalence is shared between different front-line public professionals such as healthcare workers, firefighters and public safety personnel. Importantly, the seroprevalence among these professionals is comparable to participants with other occupations, either showing symptoms or inhabiting with a COVID-19 confirmed case. The number of symptoms reported positively correlate with IgG seroprevalence. Among these symptoms, ageusia/anosmia, pneumonia and cutaneous manifestations are the three symptoms correlating with higher rates of IgG seroprevalence. However, the most frequent symptoms among IgG+ participants are fever, ageusia/anosmia and asthenia. Therefore, heterogeneity among populations should be considered when defining seroprevalence and key diagnostic symptoms.

\section{Methods}

\section{Study design and participants}

This study was performed at the laboratory facilities of Empireo Diagnóstico Molecular (www.empireo.es) in Madrid. The recruitment of the participants was conducted since April $15^{\text {th }}$ to June $15^{\text {th }}, 2020$. Written informed consent was obtained from all study participants.

Of note, this study was performed under the lockdown in Spain due to COVID-19 dictated by the Spanish government in March $14^{\text {th }}, 2020$, according to RD463/2020 ${ }^{14}$. During this period, in April $14^{\text {th }}$, the government order SND/344/2020 established the requirement for a medical prescription to be allowed to perform a diagnostic test for COVID-19 ${ }^{15}$. We adopted the more restrictive and ethical interpretation of this rule to screen for participants and therefore, to be potentially included in this study. Thus, the inclusion criteria were:

1) to have suffered symptoms compatible with COVID-19 at least, 14 days before testing to fulfill the quarantine period established by the sanitary authorities; 2 ) to have been in contact with a confirmed COVID-19 case; 3) to belong to an essential collective (those allowed to keep working during the lockdown). Exclusion criteria included lack of informed consent, or failure to contact and subsequent fulfilling out information for study records. 
The above mentioned RD463/2020 in March $14^{\text {th }}$ imposed mobility restrictions for those considered nonessential personnel, limiting the movement between towns in a first stage and between provinces in a second one. These restrictions constrain the territorial distribution of the included subjects mostly to Madrid city and province

\section{Detection of SARS-CoV-2 antibodies}

The test (REAL COVID19 Rapid test cassette; Durviz, Valencia, Spain; reference RPPCOV1925) was a lateral-flow immunochromatographic assay for qualitative detection and differentiation of IgG and IgM against SARS-CoV-2 proteins, which yields results in $10 \mathrm{~min}$. The manufacturer reports a $95 \%$ Confidence Interval $(95 \% \mathrm{Cl})$ of $86 \%-100 \%$ of sensitivity for $\operatorname{lgG}$ and $95 \% \mathrm{Cl}$ of $62.1 \%-96.8 \%$ for $\operatorname{lgM}$ and specificity of $95 \% \mathrm{Cl}: 89.4 \%-99.9 \%$ for IgG and $95 \% \mathrm{Cl}: 86.5 \%-99.5 \%$ for IgM, using RT-PCR as gold standard. No cross- reactivity was shown with samples positive for Influenza A and B, Hepatitis B, Syphilis and HIV. Serum was obtained from blood after centrifugation and assayed immediately according to manufacturer's instructions.

Due to the low seroprevalence of IgM in the cohort (around 2\%), most likely due to its short duration after infection and the time lapse between the putative infection and the test imposed as the first inclusion criteria, the results shown here are only based on IgG.

\section{Statistical analysis}

Seroprevalence of antibodies against SARS-CoV-2 was calculated as proportions with $95 \% \mathrm{Cl}$ (confidence interval). We tested the association between variables with the Fisher's exact test or Chi-square test, when two or more variables were analyzed, respectively. Univariable analyses based on Odd Ratios (OR) were run to evaluate factors associated with antibody seroprevalence. Data are provided as OR and $95 \% \mathrm{Cl}$ for each variable analyzed, giving value $\mathrm{OR}=1$ to the reference category.

The analysis was carried out using Microsoft Excel and v16.16.08 (Microsoft, Redmon, WA) and Graphpad Prism v6.0c (Graphpad software, San Diego, CA).

\section{Declarations}

\section{ACKNOWLEDMENTS}

We are extremely grateful to our participants for the generous donation of their time, samples and informed consent to include their data in this work. We thank LABIANA Pharmaceuticals SLU for offering their tests during the shortage scenario suffered during the lockdown. Thank you to uncle Juan for his insightful thoughts. C.d.F. is supported by the AECC Foundation as the recipient of an "Ayuda Fundación Científica AECC a personal investigador en cancer", and would like to offer this work to the memory of Lourdes García. The CNIC is supported by the Ministerio de Ciencia, Innovación y Universidades and the Pro CNIC Foundation, and is a Severo Ochoa Center of Excellence (SEV-2015- 0505). 


\section{AUTHOR CONTRIBUTIONS}

P.T.M, P.D.G., M.R.S. received participants and collect demographic information, obtained blood samples and performed tests. R.R.S. prescribed tests when indicated following government guidelines. F.G and M.A.LL. conceived the study. P.T.M, P.S-L and C.delF. analyzed data. P.S-L and C.delF. performed statistical analysis and wrote the manuscript. All the authors reviewed the manuscript.

\section{COMPETING INTERESTS}

M.A.LL, F.G and C.delF. are founders, board members and shareholders of EMPIREO Diagnóstico Molecular.

\section{References}

1. Mulligan, M. J. et al. Final-submission v2.0 $\mathrm{P}$ a g e | 1 Phase $1 / 2$ Study to Describe the Safety and Immunogenicity of a COVID-19 RNA Vaccine Candidate (BNT162b1) in Adults 18 to 55 Years of Age: Interim Report. medRxiv 06.30.20142570 (2020) doi:10.1101/2020.06.30.20142570.

2. Azkur, A. K. et al. Immune response to SARS- CoV- 2 and mechanisms of immunopathological changes in COVID- Allergy (2020) doi:10.1111/all.14364.

3. Li, R. et al. Substantial undocumented infection facilitates the rapid dissemination of novel coronavirus (SARS-CoV2). Science (80-. ). eabb3221 (2020) doi:10.1126/science.abb3221.

4. Young, B. E. et al. Epidemiologic Features and Clinical Course of Patients Infected with SARS-CoV-2 in Singapore. JAMA - J. Am. Med. Assoc. 323, 1488-1494 (2020).

5. Lin, L., Lu, L., Cao, W. \& Li, T. Hypothesis for potential pathogenesis of SARS-CoV-2 infection--a review of immune changes in patients with viral pneumonia. Microbes Infect. 1-14 (2020) doi:10.1080/22221751.2020.1746199.

6. Chen, G. et al. Clinical and immunological features of severe and moderate coronavirus disease 2019. Clin. Invest. 130, 2620-2629 (2020).

7. Zhou, F. et al. Clinical course and risk factors for mortality of adult inpatients with COVID-19 in Wuhan, China: a retrospective cohort study. Lancet 395, 1054-1062 (2020).

8. Huang, C. et al. Clinical features of patients infected with 2019 novel coronavirus in Wuhan, China. Lancet 395, 497-506 (2020).

9. Prather, K. A., Wang, C. C. \& Schooley, R. T. Reducing transmission of SARS-CoV-2. Science (80-. ). eabc6197 (2020) doi:10.1126/science.abc6197.

10. Britton, T., Ball, F. \& Trapman, P. A mathematical model reveals the influence of population heterogeneity on herd immunity to SARS-CoV-2. Science (2020) doi:10.1126/science.abc6810. 
11. Pollán, M. et al. Prevalence of SARS-CoV-2 in Spain (ENE-COVID): a nationwide, population-based seroepidemiological study. Lancet (London, England) (2020) doi:10.1016/S0140-6736(20)31483-5.

12. Garcia-basteiro, A. L. et al. Seroprevalence of antibodies against SARS- CoV-2 among health care workers in a large Spanish reference hospital. Commun. 11, 1-36 (2020).

13. Barallat, J., Fernández-Rivas G, -Sánchez B, Q. \& Martinez-Caceres. Seroprevalence of SARS-CoV-2 IgG Specific Antibodies among Healthcare Workers in the Northern Metropolitan Area of Barcelona, Spain, after the first pandemic wave. doi:10.1101/2020.06.24.20135673.

14. España. Ministerio de la Presidencia. Real Decreto 463/2020. Boletín Of. del Estado 67, 2539025400 (2020).

15. Jefatura del Estado, B. Disposición 3824 del BOE núm. 73 de 2020. https://www.boe.es (2020).

16. Stringhini, S. et al. Seroprevalence of anti-SARS-CoV-2 IgG antibodies in Geneva, Switzerland (SEROCoV-POP): a population-based study. Lancet313-319 (2020) doi:10.1016/S01406736(20)31304-0.

17. Sotgiu, G. et al. SARS-CoV-2 specific serological pattern in healthcare workers of an Italian COVID-19 forefront hospital. BMC Pulm. Med. 20, 203 (2020).

18. Korth, J. et al. SARS-CoV-2-specific antibody detection in healthcare workers in Germany with direct contact to COVID-19 patients. Clin. Virol. 128, (2020).

19. Pallett, S. J. C. et al. Point-of-care serological assays for delayed SARS- CoV-2 case identification among health-care workers in the UK: a prospective multicentre cohort study. Respir. Med. (2020) doi:10.1016/S2213-2600(20)30315-5.

20. Stubblefield, W. B. et al. Seroprevalence of SARS-CoV-2 Among Frontline Healthcare Personnel During the First Month of Caring for COVID-19 Patients - Nashville, Tennessee. Infect. Dis. (2020).

21. Chen, Y. et al. High SARS-CoV-2 antibody prevalence among healthcare workers exposed to COVID-19 patients. Infect. (2020) doi:10.1016/j.jinf.2020.05.067.

22. Garralda Fernández, J., Molero Vilches, I., Bermejo Rodríguez, A. \& Cano Torres, Impact of SARS-CoV2 pandemic among health care workers in a secondary teaching hospital in Spain. medRxiv (2020) doi:10.1101/2020.07.26.20162529.

23. Fischer, B., Knabbe, C. \& Vollmer, T. SARS-CoV-2 IgG seroprevalence in blood donors located in three different federal states, Germany, March to June 2020. Eurosurveillance 25, (2020).

24. Liu, X. et al. Patterns of IgG and IgM antibody response in COVID-19 patients. Emerging Microbes and Infections 9 1269-1274 (2020).

25. Li, L. et al. Characteristics and serological patterns of COVID-19 convalescent plasma donors: optimal donors and timing of donation. Transfusion 15918 (2020) doi:10.1111/trf.15918.

26. Zhao, J. et al. Antibody responses to SARS-CoV-2 in patients of novel coronavirus disease 2019. medRxiv 03.02.20030189 (2020) doi:10.1101/2020.03.02.20030189. 
27. Guo, L. et al. Profiling Early Humoral Response to Diagnose Novel Coronavirus Disease (COVID-19). Infect. Dis. (2020) doi:10.1093/cid/ciaa310.

28. Long, Q. X. et al. Antibody responses to SARS-CoV-2 in patients with COVID-19. Med. 26, 845-848 (2020).

29. Randolph, H. E. \& Barreiro, L. B. Herd Immunity: Understanding COVID- 19. Immunity 52 737-741 (2020).

30. Havers, F. P. et al. Seroprevalence of Antibodies to SARS-CoV-2 in 10 Sites in the United States, March 23-May 12, 2020. JAMA Intern. Med. (2020) doi:10.1001/jamainternmed.2020.4130.

31. Griffin, S. Covid-19: Herd immunity is "unethical and unachievable," say experts after report of $5 \%$ seroprevalence in Spain. BMJ m2728 (2020) doi:10.1136/bmj.m2728.

32. Liu, R. et al. The comparative superiority of $\lg M-\lg G$ antibody test to real- time reverse 1 transcriptase PCR detection for SARS-CoV-2 infection diagnosis 2 Corresponding authors 18. doi:10.1101/2020.03.28.20045765.

33. Vabret, N. et al. Immunology of COVID-19: Current State of the Immunity (2020) doi:10.1016/j.immuni.2020.05.002.

34. Grifoni, A. et al. Targets of T Cell Responses to SARS-CoV-2 Coronavirus in Humans with COVID-19 Disease and Unexposed Individuals. Cell (2020) doi:10.1016/j.cell.2020.05.015.

35. Ni, L. et al. Detection of SARS-CoV-2-Specific Humoral and Cellular Immunity in COVID-19 Convalescent Individuals. Immunity (2020) doi:10.1016/j.immuni.2020.04.023.

36. Braun, J. et al. SARS-CoV-2-reactive T cells in healthy donors and patients with COVID-19. Nature (2020) doi:10.1038/s41586-020-2598-9.

37. Liao, M. et al. Single-cell landscape of bronchoalveolar immune cells in patients with COVID-19. Med. 26, 842-844 (2020).

38. Yousaf, A. R. et al. A prospective cohort study in non-hospitalized household contacts with SARSCoV-2 infection: symptom profiles and symptom change over time. Infect. Dis. (2020) doi:10.1093/cid/ciaa1072.

39. Israel, A. et al. Smoking and the risk of COVID-19 in a large observational population study. doi:10.1101/2020.06.01.20118877.

40. Lynch, K. L. et al. Magnitude and kinetics of anti-SARS-CoV-2 antibody responses and their relationship to disease severity. medRxiv 06.03.20121525 (2020) doi:10.1101/2020.06.03.20121525.

41. Gavriatopoulou, M. et al. Organ-specific manifestations of COVID-19 infection. Exp. Med. (2020) doi:10.1007/s10238-020-00648-x.

42. Henry, D., Ackerman, M., Sancelme, E., Finon, A. \& Esteve, E. Urticarial eruption in COVID-19 infection. Journal of the European Academy of Dermatology and Venereology 34 e244-e245 (2020).

43. Lapostolle, F. et al. Clinical features of 1487 COVID-19 patients with outpatient management in the Greater Paris: the COVID-call study. Intern. Emerg. Med. (2020) doi:10.1007/s11739-020-02379-z. 


\section{Tables}

Table 1. Baseline characteristics of study participants.

\begin{tabular}{|c|c|c|c|}
\hline & & $n$ & $\%$ \\
\hline Total & & 449 & $100 \%$ \\
\hline \multirow{2}{*}{ Sex } & Male & 250 & 55.689 \\
\hline & Female & 199 & $44.32 \%$ \\
\hline \multirow{7}{*}{ Age (years) } & $<20$ & 14 & $3.12 \%$ \\
\hline & $20-29$ & 38 & $8.46 \%$ \\
\hline & $30-39$ & 111 & $24.72 \%$ \\
\hline & $40-49$ & 113 & 25.1796 \\
\hline & $50-59$ & 88 & $19.60 \%$ \\
\hline & $60-69$ & 64 & $14.25 \%$ \\
\hline & 270 & 21 & $4.67 \%$ \\
\hline \multirow{2}{*}{ Smoker } & No & 375 & $83.52 \%$ \\
\hline & Yes & 74 & 16.4896 \\
\hline \multirow{2}{*}{ Comorbidities $^{1}$} & No & 346 & 77.0696 \\
\hline & Yes & 103 & 22.949 \\
\hline \multirow{4}{*}{ Occupation } & Others ${ }^{2}$ & 359 & $76.96 \%$ \\
\hline & Healthcare workers & 43 & $9.58 \%$ \\
\hline & Firefighters & 18 & $4.01 \%$ \\
\hline & Police/Public safety & 29 & $6.46 \%$ \\
\hline \multirow{2}{*}{ Municipality } & Madrid area & 300 & $66.82 \%$ \\
\hline & Madrid city & 149 & $33.18 \%$ \\
\hline \multirow{3}{*}{ PCR status } & Never done & 403 & 89.7696 \\
\hline & Negative & 33 & $7.35 \%$ \\
\hline & Positive & 13 & $2.89 \%$ \\
\hline \multirow{3}{*}{$\begin{array}{l}\text { Contact with confirmed } \\
\text { case }\end{array}$} & No contact & 170 & $37.86 \%$ \\
\hline & Household member & 135 & 30.0796 \\
\hline & Non-cohabitating contact & 144 & 32.0796 \\
\hline \multirow{2}{*}{$\begin{array}{l}\text { Symptoms compatible } \\
\text { with COVID-193 }\end{array}$} & No & 137 & $30.51 \%$ \\
\hline & Yes & 312 & $69.49 \%$ \\
\hline
\end{tabular}

${ }^{1}$ Comorbidities include: vascular system and digestive tract diseases, hypothyroidism, epilepsy, diabetes, chronic respiratory disease (asthma, allergy, COPD), cancers and autoimmune disorders.

${ }_{2}^{2}$ Those patients that are not among the other three specific professions.

${ }^{3}$ Fever, headache, cough, odynophagia, asthenia, myalgia, ageusia, anosmia, dyspnea,

pneumonia, gastrointestinal symptoms and/or cutaneous manifestations. 
Table 2. Seroprevalence of SARS-CoV-2 by general characteristics.

\begin{tabular}{|c|c|c|c|c|c|c|}
\hline & & \multicolumn{2}{|c|}{ IgG Seroprevalence } & \multicolumn{3}{|c|}{ Univariable analysis } \\
\hline & & $\%$ & $(95 \% \mathrm{Cl})$ & P-value & OR & $95 \% \mathrm{Cl}$ \\
\hline \multirow{2}{*}{ Sex } & Male & 32.40 & $(26.64-38.58)$ & \multirow{2}{*}{$0.548^{1}$} & \multicolumn{2}{|l|}{1} \\
\hline & Female & 38.18 & $(28.56-42.24)$ & & 1.13 & 0.76 to 1.68 \\
\hline \multirow{7}{*}{ Age (years) } & $<20$ & 42.86 & $(17.66-71-14)$ & \multirow{7}{*}{$0.298^{2}$} & 1 & \\
\hline & $20-29$ & 34.21 & $(19.63-53.65)$ & & 0.69 & 0.20 to 2.43 \\
\hline & $30-39$ & 24.32 & (16.68-33.38) & & 0.43 & 0.14 to 1.35 \\
\hline & $40-49$ & 33.63 & $(25.01-43.12)$ & & 0.68 & 0.22 to 2.09 \\
\hline & $50-59$ & 36.36 & $(26.31-47.31)$ & & 0.76 & 0.24 to 2.39 \\
\hline & $60-69$ & 42.19 & (29.94-55.18) & & 0.97 & 0.30 to 3.13 \\
\hline & $\geq 70$ & 33.33 & (14.59-56.97) & & 0.67 & 0.17 to 2.69 \\
\hline \multirow{2}{*}{ Smoker } & No & 36.00 & (31.14-41.09) & \multirow{2}{*}{$0.021^{1}\left({ }^{*}\right)$} & 1 & \\
\hline & Yes & 21.62 & $(12.89-32.72)$ & & 0.49 & 0.27 to 0.89 \\
\hline \multirow{2}{*}{ Comorbidities } & No & 32.08 & $(27.19-37.28)$ & \multirow{2}{*}{$0.235^{1}$} & 1 & \\
\hline & Yes & 38.83 & $(29.39-48.94)$ & & 1.34 & 0.85 to 2.12 \\
\hline \multirow{4}{*}{ Occupation } & Others & 35.38 & $(30.43-40.57)$ & \multirow{4}{*}{$0.406^{2}$} & 1 & \\
\hline & Healthcare workers & 25.58 & $(13.52-41.17)$ & & 0.63 & 0.31 to 1.29 \\
\hline & Firefighters & 33.33 & (13.34-59.01) & & 0.91 & 0.33 to 2.49 \\
\hline & Police/Public safety & 24.14 & $(10.30-43.54)$ & & 0.58 & 0.24 to 1.40 \\
\hline \multirow{2}{*}{ Municipality } & Madrid area & 32.89 & $(25.42-41.05)$ & \multirow{2}{*}{$0.833^{1}$} & 1 & \\
\hline & Madrid city & 34.00 & (28.65-39.67) & & 1.05 & 0.69 to 1.59 \\
\hline
\end{tabular}

${ }^{1}$ Fisher's exact test

${ }^{2}$ Chi-square test

OR: Odds Ratio. 95\% Cl: 95\% Confidence Interval. 
Table 3. Seroprevalence of SARS-CoV-2 by self-reported clinical characteristics.

\begin{tabular}{|c|c|c|c|c|c|c|}
\hline & & \multicolumn{3}{|c|}{ IgG Seroprevalence } & \multicolumn{2}{|c|}{ Univariable analysis } \\
\hline & & $\%$ & (95\% Cl) & P-value & OR & $95 \% \mathrm{Cl}$ \\
\hline \multirow{3}{*}{ PCR status } & Never done & 29.28 & $(24.48-33.99)$ & \multirow{3}{*}{$<0.001(* * *)^{2}$} & 1 & \\
\hline & Negative & 42.42 & $(25.48-60.78)$ & & 1.78 & 0.86 to 3.67 \\
\hline & Positive & 92.31 & $(63.97-99.81)$ & & 29.98 & 3.72 to 225.50 \\
\hline \multirow{3}{*}{ Contact with confirmed case } & No contact & 24.74 & $(18.42-31.89)$ & \multirow{3}{*}{$<0.001(* * *)^{2}$} & 1 & \\
\hline & Household member & 48.15 & $(39.47-56.41)$ & & 2.83 & 1.74 to 4.60 \\
\hline & Non-cohabitating contact & 29.86 & $(22.53-38.04)$ & & 1.30 & 0.79 to 2.14 \\
\hline \multirow{2}{*}{$\begin{array}{l}\text { Symptoms compatible with } \\
\text { covi-19 }\end{array}$} & No & 14.60 & $(9.15-21.64)$ & \multirow{2}{*}{$<0.001(* * *)^{1}$} & 1 & \\
\hline & Yes & 41.99 & $(36.45-47.68)$ & & 4.29 & 2.54 to 7.26 \\
\hline \multirow{11}{*}{ - Type of symptoms } & Ageusia/Anosmia & 77.36 & $(68.21-84.92)$ & $<0.001(* * *)^{1}$ & 13.57 & 8.02 to 22.96 \\
\hline & Pneumonia & 75.00 & $(47.62-92.73)$ & $<0.001(* * *)^{1}$ & 6.35 & 2.01 to 20.03 \\
\hline & Cutaneous & 63.33 & $(43.86-80.07)$ & $0.001(* * *)^{1}$ & 3.76 & 1.74 to 8.12 \\
\hline & Fever & 47.51 & $(40.06-55.05)$ & $<0.001(* * *)^{1}$ & 2.83 & 1.89 to 4.23 \\
\hline & Gl symptoms & 50.47 & $(40.63-60.23)$ & $<0.001(* * *)^{1}$ & 2.57 & 1.65 to 4.02 \\
\hline & Asthenia & 44.51 & $(36.97-52.24)$ & $<0.001(* * *)^{1}$ & 2.19 & 1.47 to 3.27 \\
\hline & Dyspnea & 47.06 & $(34.83-59.55)$ & $0.0125\left(^{*}\right)^{1}$ & 1.96 & 1.16 to 3.30 \\
\hline & Cough & 42.67 & $(34.64-50.99)$ & $0.0041(* *)^{1}$ & 1.84 & 1.22 to 2.78 \\
\hline & Myalgia & 42.47 & (34.93-50.51) & $0.0076(* *)^{1}$ & 1.78 & 1.18 to 2.68 \\
\hline & Headache & 40.46 & $(33.08-48.18)$ & $0.0182\left({ }^{*}\right)^{1}$ & 1.64 & 1.10 to 2.44 \\
\hline & Odynophagia & 32.20 & $(23.90-41.43)$ & $0.7346^{1}$ & 0.92 & 0.59 to 1.43 \\
\hline \multirow{5}{*}{ - Number of symptoms } & 0 & 14.60 & $(9.15-21.64)$ & \multirow{5}{*}{$<0.001(* * *)^{2}$} & 1 & \\
\hline & $1-3$ & 32.59 & $(24.78-41.19)$ & & 2.83 & 1.56 to 5.13 \\
\hline & $4-6$ & 38.79 & $(29.89-48.29)$ & & 3.71 & 2.03 to 6.78 \\
\hline & $7-9$ & 70.00 & $(55.39-82.14)$ & & 13.65 & 6.33 to 29.45 \\
\hline & $\geq 10$ & 63.64 & $(30.79-89.07)$ & & 10.24 & 2.74 to 38.21 \\
\hline
\end{tabular}

${ }^{1}$ Fisher's exact test

${ }^{2}$ Chi-square test

OR, Odds Ratio. 95\% Cl, 95\% Confidence interval. GI, Gastrointestinal

\section{Figures}
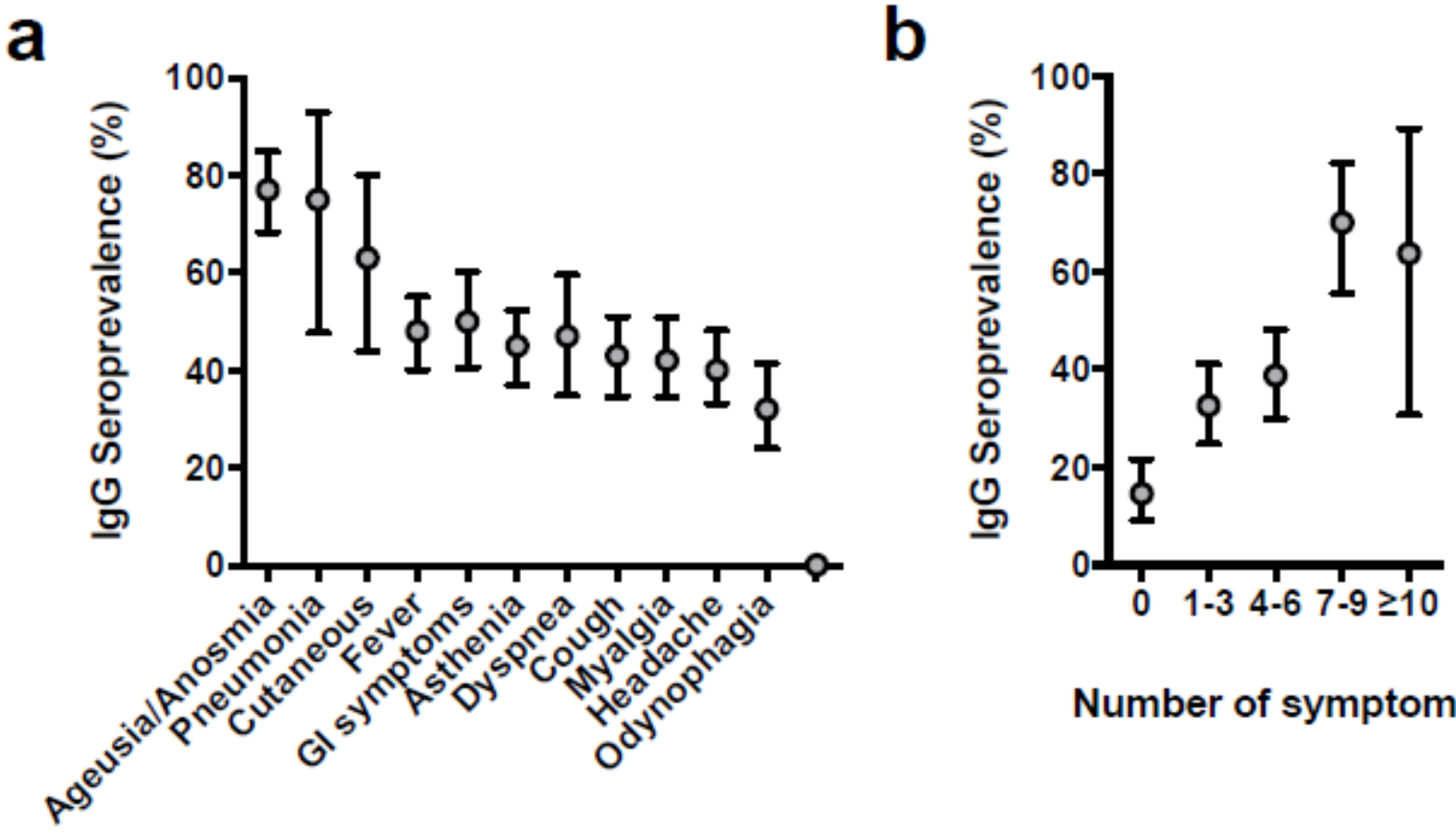

Number of symptoms 
Seroprevalence of SARS-CoV-2 by COVID-19 compatible symptoms. a) IgG seroprevalence for each of the reported symptoms. b) IgG seroprevalence grouped by number of individual symptoms reported. Dots and vertical lines represent mean and 95\% confidence interval, respectively. GI, Gastrointestinal.

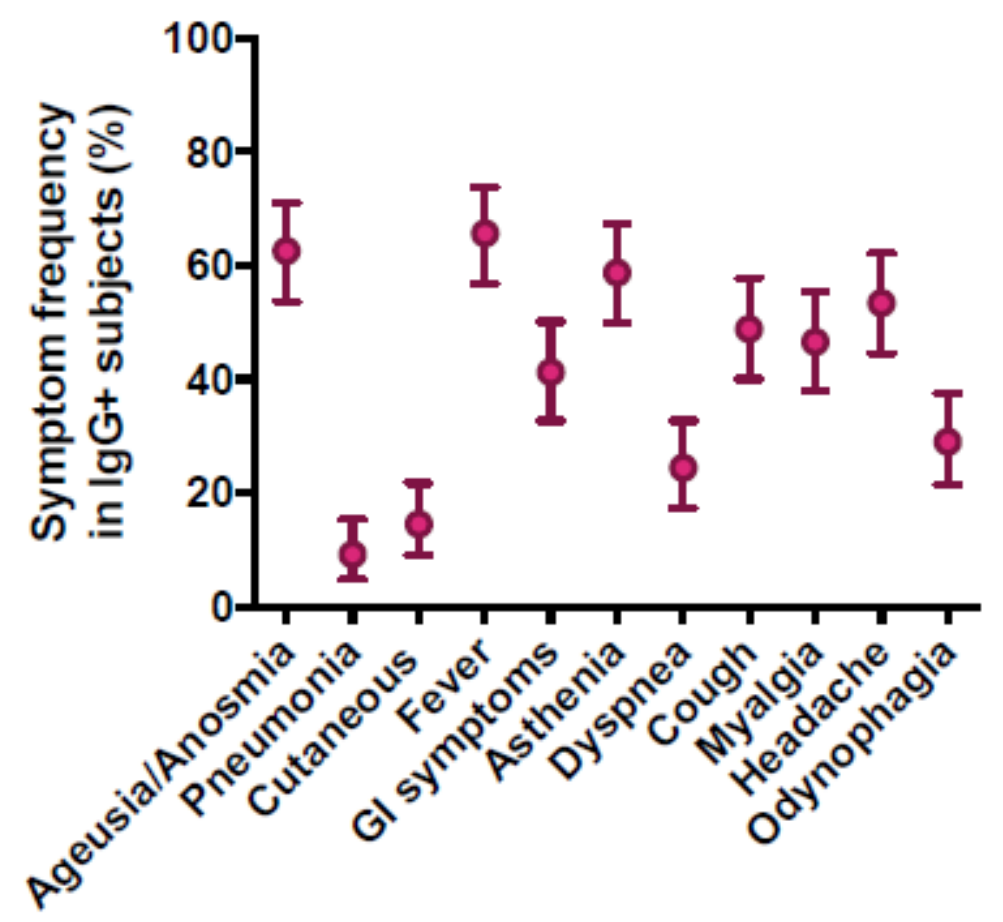

Figure 2

Symptoms distribution within IgG positive subjects. Dots and vertical lines represent mean and 95\% confidence interval, respectively. Gl, Gastrointestinal.

\section{Supplementary Files}

This is a list of supplementary files associated with this preprint. Click to download.

- TorresetalSuppFigure1.pdf 